Bull. Austral. Math. Soc.

$34 \mathrm{~A} 40,39 \mathrm{~A} 12,65 \mathrm{D} 99$

VOL. $61(2000)$ [371-385]

\title{
CONTINUOUS AND DISCRETE HALANAY-TYPE INEQUALITIES
}

\section{S. Mohamad And K. Gopalsamy}

\begin{abstract}
We consider continuous time and discrete time Halanay-type inequalities for nonautonomous scalar systems with discrete and distributed delays. The results obtained generalise the existing results of Halanay and improve certain results of Baker and Tang. Furthermore, it is shown that the discrete time inequalities which are analogues of continuous time inequalities preserve the stability conditions corresponding to the continuous time Halanay-type inequalities.
\end{abstract}

\section{INTRODUCTION}

Differential inequalities have played a significant role in the analysis of continuous and discrete time dynamical systems and elsewhere in nonlinear analysis (Halanay [5], Lakshmikantham and Leela [6, 7], Walter [8], Agarwal [4]). Halanay [5, p.378] studied the stability of the zero solution of

$$
\frac{d u(t)}{d t}=a u(t)+b u(t-\tau), \quad t>t_{0}, \quad \tau>0
$$

by using a differential inequality in which the stability of the zero solution of (1.1) is related to that of the zero solution of

$$
\frac{d u(t)}{d t}=(a+b) u(t) \quad \text { when } \quad a+b<0 .
$$

The following result has been used by Gopalsamy [4] in the derivation of sufficient conditions for the stability of linear delay differential equations.

Lemma 1.1. Let $v(t)>0, t \in \mathbb{R}$ and $\tau \in[0, \infty), t_{0} \in \mathbb{R}$. Suppose

$$
v^{\prime}(t) \leqslant-a v(t)+b\left(\sup _{t-\tau \leqslant s \leqslant t} v(s)\right), \quad t>t_{0} .
$$

If $a>b>0$ then there exist constants $\gamma>0$ and $k>0$ such that $v(t) \leqslant k e^{-\gamma\left(t-t_{0}\right)}$ for $t>t_{0}$ and hence $v(t) \rightarrow 0$ as $t \rightarrow \infty$.

Received 13th July, 1999

The first author was on leave from Department of Mathematics, University of Brunei Darussalam, Bandar Seri Begawan BE 1410, Brunei Darussalam.

Copyright Clearance Centre, Inc. Serial-fee code: 0004-9727/00 \$A2.00+0.00. 
Hereafter $v^{\prime}(t)$ as in (1.2) denotes the upper right derivative. Baker and Tang [2] have considered a nonautonomous version of (1.2) and obtained a special case of a Halanaytype inequality. In particular they have established the following result.

Lemma 1.2. Let $v(t)>0, t \in \mathbb{R}$ and let $a(\cdot), b(\cdot)$ be continuous on $\mathbb{R}$. Suppose further that

$$
\begin{aligned}
& v^{\prime}(t) \leqslant-a(t) v(t)+b(t)\left(\sup _{q(t) \leqslant s \leqslant t} v(s)\right), \quad t>t_{0} \\
& v(t)=|\varphi(t)| \text { for } t \leqslant t_{0}
\end{aligned}
$$

where $\varphi(t)$ is bounded and continuous for $t \leqslant t_{0}, a(t) \geqslant 0, b(t) \geqslant 0$ for $t \in\left[t_{0}, \infty\right)$, $q(t) \leqslant t$ and $q(t) \rightarrow \infty$ as $t \rightarrow \infty$. If there exists $\sigma>0$ such that

$$
-a(t)+b(t) \leqslant-\sigma<0 \text { for } t \geqslant t_{0}
$$

then

$$
\text { (i) } v(t) \leqslant\|\varphi\|^{\left(-\infty, t_{0}\right]}, \quad t \geqslant t_{0} \quad \text { and } \quad \text { (ii) } v(t) \rightarrow 0 \text { as } t \rightarrow \infty
$$

where $\|\varphi\|^{\left(-\infty, t_{0}\right]}=\sup _{t \in\left(-\infty, t_{0}\right]}|\varphi(t)|<\infty$.

We note that discrete time versions of Halanay-type inequalities with finite and distributed delays have also been considered by Baker and Tang [2]. However, the discrete versions considered by Baker and Tang [2] do not provide sufficient conditions for uniform asymptotic stability obtained from the continuous time mother versions.

In this paper we consider nonautonomous continuous time Halanay-type inequalities with finite and distributed delays. It will be shown that our results generalise the existing results of Halanay [5, p.378] and improve those results of Baker and Tang [2]. Moreover, we also consider discrete time analogues of the continuous time Halanay-type inequalities; it is shown that our discrete time analogues preserve the stability conditions of their continuous time counterparts. Sufficient conditions are obtained which also preserve the convergence characteristics of solutions satisfying both types of inequalities.

\section{Continuous Halanay-type inEqualities}

In this section we consider continuous time Halanay-type inequalities with finite and distributed delays in nonautonomous delay differential inequalities. First we provide results for the continuous time Halanay-type inequality with finite delays.

THEOREM 2.1. Let $x(\cdot)$ be a nonnegative function satisfying

$$
\begin{aligned}
\frac{d x(t)}{d t} & \leqslant-a(t) x(t)+b(t)\left(\sup _{t-\tau(t) \leqslant s \leqslant t} x(s)\right), \quad t>t_{0} \\
x(s) & =|\varphi(s)| \quad \text { for } \quad s \in\left[t_{0}-\tau^{*}, t_{0}\right]
\end{aligned}
$$


where $\tau(t)$ denotes a nonnegative, continuous and bounded function defined for $t \in \mathbb{R}$ and $\tau^{*}=\sup _{t \in \mathbb{R}} \tau(t) ; \varphi(s)$ is continuous and defined for $s \in\left[t_{0}-\tau^{*}, t_{0}\right] ; a(t)$ and $b(t)$, $t \in \mathbb{R}$, denote nonnegative, continuous and bounded functions. Suppose

$$
a(t)-b(t) \geqslant \sigma, \quad t \in \mathbb{R}
$$

where $\sigma=\inf _{t \in \mathbf{R}}(a(t)-b(t))>0$. Then there exists a positive number $\tilde{\mu}$ such that

$$
x(t) \leqslant\left(\sup _{s \in\left[t_{0}-\tau^{*}, t_{0}\right]} x(s)\right) e^{-\tilde{\mu}\left(t-t_{0}\right)}, \quad t>t_{0} .
$$

Proof: We define the function $G$ as follows:

$$
G(t, \mu)=-a(t)+\mu+b(t) e^{\mu \tau^{*}}, \quad \mu \in \mathbb{R}, \quad t \in \mathbb{R} .
$$

Since $a(t)$ and $b(t)$ are continuous and bounded for $t \in \mathbb{R}$ we consider the function $F$ defined by

$$
F(\mu)=\sup _{t \in \mathbb{R}} G(t, \mu), \quad \mu \in \mathbb{R} .
$$

Clearly, $F$ is a continuous function of $\mu \in \mathbb{R}$. By applying the assumption (2.3) we have from (2.6) that

$$
F(0)=\sup _{t \in \mathbb{R}} G(t, 0)=\sup _{t \in \mathbb{R}}(-a(t)+b(t))=-\inf _{t \in \mathbb{R}}(a(t)-b(t))=-\sigma<0 .
$$

Moreover, by the boundedness of $a(\cdot)$ and $b(\cdot)$ we have

$$
-a^{*}+\mu+b_{*} e^{\mu \tau^{*}}=F_{*}(\mu) \leqslant F(\mu) \leqslant F^{*}(\mu)=-a_{*}+\mu+b^{*} e^{\mu \tau^{*}}, \quad \mu \in \mathbb{R}
$$

where

$$
a_{*}=\inf _{t \in \mathbf{R}} a(t), \quad a^{*}=\sup _{t \in \mathbb{R}} a(t), \quad b_{*}=\inf _{t \in \mathbb{R}} b(t) \quad \text { and } \quad b^{*}=\sup _{t \in \mathbb{R}} b(t) .
$$

We observe that $F_{*}(\mu) \rightarrow \infty$ and $F^{*}(\mu) \rightarrow \infty$ monotonically as $\mu \rightarrow \infty$ and hence from (2.8) we deduce that

$$
F(\mu)=\sup _{t \in \mathbb{R}} G(t, \mu) \rightarrow \infty \text { monotonically as } \mu \rightarrow \infty .
$$

Now let $0<\tilde{\sigma}<\sigma$. It follows from (2.7), (2.9) and the continuity of $F(\mu)$ that there exists a number $\tilde{\mu}>0$ such that

$$
F(\tilde{\mu})=\sup _{t \in \mathbf{R}} G(t, \tilde{\mu})=-\tilde{\sigma}<0 \text { where } \quad 0<\tilde{\sigma}<\sigma,
$$


and this implies that

$$
-a(t)+\tilde{\mu}+b(t) e^{\tilde{\mu} \tau^{\bullet}} \leqslant-\tilde{\sigma}<0 \text { for all } t \in \mathbb{R} .
$$

Now we define

$$
\widetilde{x}(t)= \begin{cases}x(t) e^{\tilde{\mu}\left(t-t_{0}\right)}, & t>t_{0} \\ x(t), & t_{0}-\tau^{*} \leqslant t \leqslant t_{0} .\end{cases}
$$

We have from (2.12) and (2.1),

$$
\begin{aligned}
\frac{d \widetilde{x}(t)}{d t} & =\frac{d x(t)}{d t} e^{\tilde{\mu}\left(t-t_{0}\right)}+x(t) \tilde{\mu} e^{\tilde{\mu}\left(t-t_{0}\right)}, \quad t>t_{0} \\
& \leqslant\left\{-a(t) x(t)+b(t)\left(\sup _{t-\tau^{*} \leqslant s \leqslant t} x(s)\right)\right\} e^{\tilde{\mu}\left(t-t_{0}\right)}+x(t) \tilde{\mu} e^{\tilde{\mu}\left(t-t_{0}\right)}, \quad t>t_{0} \\
& \leqslant(-a(t)+\widetilde{\mu}) \widetilde{x}(t)+b(t) e^{\tilde{\mu} \tau^{*}}\left(\sup _{t-\tau^{*} \leqslant s \leqslant t} \widetilde{x}(s)\right), \quad t>t_{0} .
\end{aligned}
$$

Since $\varphi(t)$ is continuous and defined for $t \in\left[t_{0}-\tau^{*}, t_{0}\right]$, we let

$$
\sup _{t \in\left[t_{0}-\tau^{*}, t_{0}\right]}|\varphi(t)|=M, \quad M>0 .
$$

Let $\delta>1$ be arbitrary. From (2.2) and (2.12) we have $\tilde{x}(t)<\delta M$ for $t \in\left[t_{0}-\tau^{*}, t_{0}\right]$. We claim

$$
\widetilde{x}(t)<\delta M \text { for } t>t_{0} \text {. }
$$

Suppose (2.14) does not hold. Let $t_{1}>t_{0}$ be the first time for which

$$
\widetilde{x}(t)<\delta M \quad \text { for } \quad t_{0}-\tau^{*} \leqslant t<t_{1}, \quad \widetilde{x}\left(t_{1}\right)=\delta M \quad \text { and } \quad \frac{d \widetilde{x}\left(t_{1}\right)}{d t} \geqslant 0 .
$$

We have from (2.13) and (2.15),

$$
\begin{aligned}
0 \leqslant \frac{d \widetilde{x}\left(t_{1}\right)}{d t} & \leqslant\left(-a\left(t_{1}\right)+\tilde{\mu}\right) \tilde{x}\left(t_{1}\right)+b\left(t_{1}\right) e^{\tilde{\mu} \tau^{*}}\left(\sup _{t_{1}-\tau^{*} \leqslant s \leqslant t_{1}} \tilde{x}(s)\right) \\
& \leqslant\left(-a\left(t_{1}\right)+\tilde{\mu}+b\left(t_{1}\right) e^{\tilde{\mu} \tau^{*}}\right) \delta M \\
& \leqslant-\tilde{\sigma} \delta M<0 \quad \text { (due to }(2.11))
\end{aligned}
$$

from which we have a contradiction. Hence the claim (2.14) holds. Since $\delta>1$ is arbitrary, by allowing $\delta \rightarrow 1_{+}$we have $\tilde{x}(t) \leqslant M$ for $t>t_{0}$. It then follows from (2.12) and the definition of $\tilde{\mu}$ that $x(t) \leqslant M e^{-\tilde{\mu}\left(t-t_{0}\right)}$ for $t>t_{0}$ and hence the assertion (2.4) is satisfied. This completes the proof.

We consider in the following an inequality with delays distributed over an unbounded interval $[0, \infty)$. 
THEDREM 2.2. Let $x(\cdot)$ be a nonnegative function satisfying

$$
\begin{aligned}
\frac{d x(t)}{d t} & \leqslant-a(t) x(t)+b(t) \int_{0}^{\infty} K(s) x(t-s) d s, \quad t>t_{0} \\
x(s) & =|\varphi(s)| \text { for } s \in\left(-\infty, t_{0}\right]
\end{aligned}
$$

where $\varphi(s)$ defined for $s \in\left(-\infty, t_{0}\right]$ is continuous and $\sup _{s \in\left(-\infty, t_{0}\right]}|\varphi(s)|=M, M>0 ; a(t)$ and $b(t)$ are defined for $t \in \mathbb{R}$ and denote nonnegative, continuous and bounded functions; the delay kernel $K(\cdot)$ is assumed to satisfy the following properties

$$
K:[0, \infty) \mapsto[0, \infty) \text { and } \int_{0}^{\infty} K(s) e^{\mu s} d s<\infty
$$

for some positive number $\mu$. Suppose further that

$$
a(t)-b(t) \int_{0}^{\infty} K(s) d s \geqslant \sigma, \quad t \in \mathbb{R}
$$

where $\sigma=\inf _{t \in \mathbb{R}}\left(a(t)-b(t) \int_{0}^{\infty} K(s) d s\right)>0$. Then there exists a positive number $\tilde{\mu}$ such that

$$
x(t) \leqslant\left(\sup _{s \in\left(-\infty, t_{0}\right]} x(s)\right) e^{-\tilde{\mu}\left(t-t_{0}\right)}, \quad t>t_{0} .
$$

Proof: It follows from (2.19) that we can find a critical value $\mu^{*}>0$ such that

$$
\int_{0}^{\infty} K(s) e^{\mu s} d s<\infty \text { for } 0<\mu<\mu^{*}
$$

For example, let $K(s)=e^{-s}$ for $s \in[0, \infty)$ and we have $\int_{0}^{\infty} e^{-s} e^{\mu s} d s=1 /(1-\mu)$ for which the critical value is $\mu^{*}=1$. Along with this observation we define the function $G(t, \mu)$ by

$$
G(t, \mu)=-a(t)+\mu+b(t) \int_{0}^{\infty} K(s) e^{\mu s} d s, \quad t \in \mathbb{R}, \quad \mu \in\left(-\infty, \mu^{*}\right)
$$

Since $a(t)$ and $b(t)$ are continuous and bounded for $t \in \mathbb{R}$ we consider the function $F$ defined by

$$
F(\mu)=\sup _{t \in \mathbf{R}} G(t, \mu) \text { for } \mu \in\left(-\infty, \mu^{*}\right)
$$

Clearly, $F(\mu)$ is continuous and defined for $\mu \in\left(-\infty, \mu^{*}\right)$. By applying the assumption (2.20) we have from (2.23) and (2.24) that

$$
\begin{aligned}
F(0) & =\sup _{t \in \mathbf{R}}\left(-a(t)+b(t) \int_{0}^{\infty} K(s) d s\right) \\
& =-\inf _{t \in \mathbb{R}}\left(a(t)-b(t) \int_{0}^{\infty} K(s) d s\right)=-\sigma<0
\end{aligned}
$$


We also observe that

$$
\begin{aligned}
-a^{*}+\mu+b_{*} \int_{0}^{\infty} & K(s) e^{\mu s} d s=F_{*}(\mu) \leqslant F(\mu) \leqslant F^{*} \\
& =-a_{*}+\mu+b^{*} \int_{0}^{\infty} K(s) e^{\mu s} d s \text { for } \mu \in\left(-\infty, \mu^{*}\right)
\end{aligned}
$$

and $F_{*}(\mu) \rightarrow \infty$ and $F^{*}(\mu) \rightarrow \infty$ monotonically as $\mu \rightarrow \mu_{-}^{*}$. Hence

$$
F(\mu)=\sup _{t \in \mathbb{R}} G(t, \mu) \rightarrow \infty \text { monotonically as } \mu \rightarrow \mu_{-}^{*} .
$$

Now let $0<\tilde{\sigma}<\sigma$. By (2.25), (2.27) and the continuity of $F(\mu)$ there exists a number $\tilde{\mu}$ satisfying $0<\widetilde{\mu}<\mu^{*}$ for which

$$
F(\tilde{\mu})=\sup _{t \in \mathbb{R}} G(t, \tilde{\mu})=-\tilde{\sigma}<0 \text { where } 0<\tilde{\sigma}<\sigma .
$$

Hence it follows that

$$
-a(t)+\widetilde{\mu}+b(t) \int_{0}^{\infty} K(s) e^{\tilde{\mu} s} d s \leqslant-\widetilde{\sigma}<0 \text { for all } t \in \mathbb{R} .
$$

Now we define

$$
\widetilde{x}(t)= \begin{cases}x(t) e^{\tilde{\mu}\left(t-t_{0}\right)}, & t>t_{0} \\ x(t), & -\infty<t \leqslant t_{0}\end{cases}
$$

We have from (2.30) and (2.17),

$$
\begin{aligned}
\frac{d \widetilde{x}(t)}{d t} & =\frac{d x(t)}{d t} e^{\tilde{\mu}\left(t-t_{0}\right)}+x(t) \widetilde{\mu} e^{\tilde{\mu}\left(t-t_{0}\right)}, \quad t>t_{0} \\
& \leqslant\left\{-a(t) x(t)+b(t) \int_{0}^{\infty} K(s) x(t-s) d s\right\} e^{\tilde{\mu}\left(t-t_{0}\right)}+x(t) \tilde{\mu} e^{\tilde{\mu}\left(t-t_{0}\right)}, \quad t>t_{0} \\
& =(-a(t)+\tilde{\mu}) \widetilde{x}(t)+b(t) \int_{0}^{\infty} K(s) e^{\tilde{\mu} s} \widetilde{x}(t-s) d s, \quad t>t_{0} .
\end{aligned}
$$

Let $\delta>1$ be arbitrary. We have from (2.18) and (2.30) that $\tilde{x}(t)<\delta M$ for $t \in\left(-\infty, t_{0}\right]$. We claim

$$
\tilde{x}(t)<\delta M \text { for } t>t_{0}
$$

Suppose (2.32) does not hold. Let $t_{1}>t_{0}$ be the first instant for which

$$
\widetilde{x}(t)<\delta M \quad \text { for } \quad-\infty<t<t_{1}, \quad \tilde{x}\left(t_{1}\right)=\delta M \quad \text { and } \quad \frac{d \tilde{x}\left(t_{1}\right)}{d t} \geqslant 0 .
$$


It then follows from (2.31) and (2.33) that

$$
\begin{aligned}
0 \leqslant \frac{d \widetilde{x}\left(t_{1}\right)}{d t} & \leqslant\left(-a\left(t_{1}\right)+\widetilde{\mu}\right) \tilde{x}\left(t_{1}\right)+b\left(t_{1}\right) \int_{0}^{\infty} K(s) e^{\tilde{\mu} s} \widetilde{x}\left(t_{1}-s\right) d s \\
& \leqslant\left(-a\left(t_{1}\right)+\widetilde{\mu}\right) \widetilde{x}\left(t_{1}\right)+b\left(t_{1}\right) \int_{0}^{\infty} K(s) e^{\tilde{\mu} s} d s\left(\sup _{s \in\left(-\infty, t_{1}\right]} \widetilde{x}(s)\right) \\
& \leqslant\left(-a\left(t_{1}\right)+\widetilde{\mu}+b\left(t_{1}\right) \int_{0}^{\infty} K(s) e^{\tilde{\mu} s} d s\right) \delta M \\
& \leqslant-\widetilde{\sigma} \delta M<0 \quad \text { (due to }(2.29))
\end{aligned}
$$

and this leads to a contradiction. Hence the claim (2.32) holds. Since $\delta>1$ is arbitrary, by allowing $\delta \rightarrow 1_{+}$we have $\widetilde{x}(t) \leqslant M$ for $t>t_{0}$. It then follows from (2.30) that $x(t) \leqslant M e^{-\tilde{\mu}\left(t-t_{0}\right)}$ for $t>t_{0}$ and hence the assertion (2.21) is satisfied. This completes the proof.

\section{Discrete halanay-Type inequalities}

While discrete time dynamical systems are important in their own right, such systems often arise in the numerical solutions and computer simulations of continuous time systems. While there are a plethora of methods by which discrete time analogues of continuous time systems can be obtained, the asymptotic behaviour of the two types of systems do not often coincide. For simulation purposes, it is important that a discrete analogue faithfully inherits the characteristics of the continuous time parent system. It is with this intention that we have obtained discrete analogues of continuous systems in this section.

We consider a discrete time analogue of the continuous time Halanay-type inequality (2.1). While there is no unique way of obtaining a discrete time system from its continuous time counterpart we approximate the inequality (2.1) in each disjoint interval of the form $t \in[n h,(n+1) h), n \in \mathbb{Z}$ where $\mathbb{Z}$ denotes the set of integers and $h$ is a finite positive real number denoting a uniform discretisation step size. We consider an approximation given by

$$
\begin{aligned}
\frac{d x(t)}{d t} & \leqslant-a\left(\left[\frac{t}{h}\right] h\right) x(t)+b\left(\left[\frac{t}{h}\right] h\right)\left(\sup _{[t / h]-[\tau(\cdot) / h] \leqslant[s / h] \leqslant[t / h]} x\left(\left[\frac{s}{h}\right] h\right)\right), \\
t & \in[n h,(n+1) h), \quad n \geqslant n_{0}, \quad n_{0} \in \mathbb{Z}
\end{aligned}
$$

where $\tau(\cdot)=\tau([t / h] h)$ and the interval $n \geqslant n_{0}$ denotes $n \in\left\{n_{0}, n_{0}+1, n_{0}+2, \ldots\right\}$. Equation (3.1) is known as an equation with piecewise constant arguments in which $[r]$ denotes the integer part of the real number $r$. Equations with piecewise constant arguments have been used by Cooke and Györi [3] in numerically approximating solutions 
of delay differential equations on an infinite interval. By applying a semi-implicit Eulertype scheme to (3.1) we obtain

$$
\begin{aligned}
& \frac{x\left(\left[\frac{t}{h}\right] h+h\right)-x\left(\left[\frac{t}{h}\right] h\right)}{h} \leqslant-a\left(\left[\frac{t}{h}\right] h\right) x\left(\left[\frac{t}{h}\right] h+h\right) \\
&+b\left(\left[\frac{t}{h}\right] h\right)\left(\sup _{[t / h]-[\tau(\cdot) / h] \leqslant[s / h] \leqslant[t / h]} x\left(\left[\frac{s}{h}\right] h\right)\right), \\
& t \in[n h,(n+1) h), \quad n \geqslant n_{0}
\end{aligned}
$$

and after some simplification we have

$$
x(n+1) \leqslant \frac{1}{1+a(n) h} x(n)+\frac{b(n) h}{1+a(n) h}\left(\sup _{n-\kappa(n) \leqslant j \leqslant n} x(j)\right), \quad n \geqslant n_{0}
$$

where we let $[t / h]=n,[s / h]=j,[\tau(\cdot) / h]=\kappa(\cdot)$ and we have used the notation $f(n)=$ $f(n h)$. The variables $n$ and $j$ are integers. The parameters $a(n)$ and $b(n)$ denote real valued, nonnegative and bounded sequences defined for $n \in \mathbb{Z}$ while the delay parameter $\kappa(n)$ defined for $n \in \mathbb{Z}$ denotes an integer valued, nonnegative, and bounded sequence. The initial values associated with (3.2) are given by

$$
x(n)=|\varphi(n)|, \quad n \in\left[n_{0}-\kappa^{*}, n_{0}\right]
$$

where $\kappa^{*}=\sup _{n \in \mathbf{Z}} \kappa(n)$ is a positive integer and the interval $\left[n_{0}-\kappa^{*}, n_{0}\right]$ denotes $\left\{n_{0}-\right.$ $\left.\kappa^{*}, n_{0}-\kappa^{*}+1, \ldots, n_{0}\right\}$. In the next result we provide results for the discrete time Halanay-type inequality with finite delays.

TheOREM 3.1. Let $h>0$ and let $x(\cdot)$ be a nonnegative sequence satisfying

$$
\begin{gathered}
x(n+1) \leqslant \frac{1}{1+a(n) h} x(n)+\frac{b(n) h}{1+a(n) h}\left(\sup _{n-\kappa(n) \leqslant j \leqslant n} x(j)\right), n \geqslant n_{0} \\
x(n)=|\varphi(n)| \text { for } n \in\left[n_{0}-\kappa^{*}, n_{0}\right]
\end{gathered}
$$

where $\kappa(n)$ denotes an integer valued, nonnegative and bounded sequence defined for $n \in \mathbb{Z}$ and $\kappa^{*}=\sup _{n \in \mathbb{Z}} \kappa(n)$ is a positive integer; $\varphi(n)$ is a real valued sequence defined for $n \in\left[n_{0}-\kappa^{*}, n_{0}\right]$; the parameters $a(n)$ and $b(n)$ defined for $n \in \mathbb{Z}$ denote real valued, nonnegative and bounded sequences. Suppose

$$
a(n)-b(n) \geqslant \sigma, \quad n \in \mathbb{Z}
$$

where $\sigma=\inf _{n \in \mathbf{Z}}(a(n)-b(n))>0$. Then there exists a real number $\tilde{\lambda}>1$ such that

$$
x(n) \leqslant\left(\sup _{n_{0}-\kappa \cdot \leqslant j \leqslant n_{0}} x(j)\right)\left(\frac{1}{\bar{\lambda}}\right)^{n-n_{0}}, n>n_{0} .
$$


Proof: Define a real valued function $G$ as follows:

$$
G(n, \lambda)=\frac{\lambda}{1+a(n) h}+\frac{b(n) h}{1+a(n) h} \lambda^{\kappa^{*}+1}-1, \quad n \in \mathbb{Z}, \quad \lambda>0 .
$$

Since $a(n)$ and $b(n)$ are bounded and nonnegative for $n \in \mathbb{Z}$, we can consider the function $F$ defined by

$$
F(\lambda)=\sup _{n \in \mathbf{Z}} G(n, \lambda), \quad \lambda>0 .
$$

Clearly, $F$ is continuous for $\lambda>0$. By applying the assumption (3.5) we have

$$
\begin{aligned}
F(1) & =\sup _{n \in \mathbf{Z}}\left(\frac{1}{1+a(n) h}+\frac{b(n) h}{1+a(n) h}-1\right) \\
& =-\inf _{n \in \mathbf{Z}}\left(\frac{h[a(n)-b(n)]}{1+a(n) h}\right) \leqslant-\frac{\sigma h}{1+a^{*} h}<0
\end{aligned}
$$

since $\sigma>0, h>0$ and $a^{*}=\sup _{n \in \mathbf{Z}} a(n)>0$. Moreover, by the boundedness of $a(\cdot)$ and $b(\cdot)$ we have

$$
F_{*}(\lambda) \leqslant F(\lambda) \leqslant F^{*}(\lambda) \text { for } \lambda>0
$$

where

$$
\begin{gathered}
F_{*}(\lambda)=\frac{\lambda}{1+a^{*} h}+\frac{b_{*} h}{1+a^{*} h} \lambda^{\kappa^{*}+1}-1, \quad \lambda>0, \\
F^{*}(\lambda)=\frac{\lambda}{1+a_{*} h}+\frac{b^{*} h}{1+a_{*} h} \lambda^{\kappa^{*}+1}-1, \quad \lambda>0, \\
a_{*}=\inf _{n \in \mathbf{Z}} a(n), \quad a^{*}=\sup _{n \in \mathbf{Z}} a(n), \quad b_{*}=\inf _{n \in \mathbf{Z}} b(n), \quad b^{*}=\sup _{n \in \mathbf{Z}} b(n) .
\end{gathered}
$$

We observe that $F_{*}(\lambda) \rightarrow \infty$ and $F^{*}(\lambda) \rightarrow \infty$ as $\lambda \rightarrow \infty$ and hence from (3.10) we deduce that

$$
F(\lambda)=\sup _{n \in \mathbf{Z}} G(n, \lambda) \rightarrow \infty \quad \text { as } \quad \lambda \rightarrow \infty .
$$

Let $0<\tilde{\sigma}<\sigma$. From (3.9), (3.11) and the continuity of $F(\lambda)$, there exists a number $\tilde{\lambda}>1$ such that

$$
F(\tilde{\lambda})=\sup _{n \in \mathbf{Z}}\left(\frac{\tilde{\lambda}}{1+a(n) h}+\frac{b(n) h}{1+a(n) h} \tilde{\lambda}^{\kappa+1}-1\right) \leqslant-\frac{\tilde{\sigma} h}{1+a^{*} h}<0
$$

and this implies that

$$
\frac{\tilde{\lambda}}{1+a(n) h}+\frac{b(n) h}{1+a(n) h} \tilde{\lambda}^{\kappa^{*}+1} \leqslant 1-\frac{\tilde{\sigma} h}{1+a^{*} h}=\rho \text { for all } n \in \mathbb{Z}
$$


where the constant $\rho$ satisfies $0<\rho<1$ since $0<\widetilde{\sigma}<\sigma<a^{*}$.

Now we define

$$
\tilde{x}(n)= \begin{cases}x(n) \tilde{\lambda}^{n-n_{0}}, & n>n_{0} \\ x(n), & n_{0}-\kappa^{*} \leqslant n \leqslant n_{0} .\end{cases}
$$

We have from (3.13) and (3.3) that

$$
\begin{aligned}
\widetilde{x}(n+1) & =x(n+1) \tilde{\lambda}^{n+1-n_{0}}, \quad n \geqslant n_{0} \\
& \leqslant\left\{\frac{1}{1+a(n) h} x(n)+\frac{b(n) h}{1+a(n) h}\left(\sup _{n-\kappa^{*} \leqslant j \leqslant n} x(j)\right)\right\} \tilde{\lambda}^{n+1-n_{0}}, \quad n \geqslant n_{0} \\
& \leqslant \frac{\tilde{\lambda}}{1+a(n) h} \widetilde{x}(n)+\frac{b(n) h}{1+a(n) h} \tilde{\lambda}^{\kappa^{*}+1}\left(\sup _{n-\kappa^{*} \leqslant j \leqslant n} \widetilde{x}(j)\right), \quad n \geqslant n_{0} .
\end{aligned}
$$

Let $\delta>1$ be arbitrary. Since $\varphi(n)$ in (3.4) is defined for $n \in\left[n_{0}-\kappa^{*}, n_{0}\right]$, we let

$$
\sup _{n \in\left[n_{0}-\kappa^{*}, n_{0}\right]}|\varphi(n)|=M, \quad M>0 .
$$

It follows from (3.4) and (3.13) that $\tilde{x}(n)<\delta M$ for $n \in\left[n_{0}-\kappa^{*}, n_{0}\right]$. We claim

$$
\tilde{x}(n)<\delta M \text { for } n>n_{0} .
$$

Suppose (3.15) does not hold. Let the integer $n_{1}>n_{0}$ (the first time) be such that

$$
\widetilde{x}(n)<\delta M \quad \text { for } n_{0}-\kappa^{*} \leqslant n<n_{1} \text { and } \widetilde{x}\left(n_{1}\right) \geqslant \delta M .
$$

We have from (3.14) and (3.16) that

$$
\begin{aligned}
\delta M & \leqslant \tilde{x}\left(n_{1}\right) \\
& \leqslant \frac{\tilde{\lambda}}{1+a\left(n_{1}-1\right) h} \widetilde{x}\left(n_{1}-1\right)+\frac{b\left(n_{1}-1\right) h}{1+a\left(n_{1}-1\right) h} \tilde{\lambda}^{\kappa^{*}+1}\left(\sup _{n_{1}-1-\kappa^{*} \leqslant j \leqslant n_{1}-1} \tilde{x}(j)\right) \\
& <\left(\frac{\tilde{\lambda}}{1+a\left(n_{1}-1\right) h}+\frac{b\left(n_{1}-1\right) h}{1+a\left(n_{1}-1\right) h} \tilde{\lambda}^{\kappa^{*}+1}\right) \delta M \\
(3.17) \quad & \leqslant \rho \delta M<\delta M \quad \text { (due to (3.12)) }
\end{aligned}
$$

and this is a contradiction. Hence the claim (3.15) holds. Since $\delta>1$ is arbitrary, by allowing $\delta \rightarrow 1_{+}$we have $\widetilde{x}(n) \leqslant M$ for $n>n_{0}$. It then follows from (3.13) that $x(n) \leqslant M(1 / \widetilde{\lambda})^{n-n_{0}}$ for $n>n_{0}$ and hence the assertion (3.6) is satisfied. This completes the proof.

We consider a discrete time analogue of the continuous time inequality with distributed delays given by (2.17). By following a similar process of discretisation as before, 
we first approximate (2.17) by an equation with piecewise constant arguments given by

$$
\begin{aligned}
& \frac{d x(t)}{d t} \leqslant-a\left(\left[\frac{t}{h}\right] h\right) x(t)+b\left(\left[\frac{t}{h}\right] h\right) \sum_{[s / h]=1}^{\infty} \mathcal{K}\left(\left[\frac{s}{h}\right] h\right) x\left(\left[\frac{t}{h}\right] h-\left[\frac{s}{h}\right] h\right), \\
& t \in[n h,(n+1) h), \quad s \in[j h,(j+1) h), \quad n \geqslant n_{0}, \quad j \in\{1,2,3, \ldots\}
\end{aligned}
$$

where $h>0$ denotes a uniform discretisation step size and $[r]$ denotes the integer part of the real number $r$. By applying a semi-implicit Euler-type scheme to (3.18) we obtain

$$
\begin{aligned}
& \frac{x\left(\left[\frac{t}{h}\right] h+h\right)-x\left(\left[\frac{t}{h}\right] h\right)}{h} \\
& \leqslant-a\left(\left[\frac{t}{h}\right] h\right) x\left(\left[\frac{t}{h}\right] h+h\right)+b\left(\left[\frac{t}{h}\right] h\right) \sum_{\mid s / h]=1}^{\infty} \mathcal{K}\left(\left[\frac{s}{h}\right] h\right) x\left(\left[\frac{t}{h}\right] h-\left[\frac{s}{h}\right] h\right), \\
& \quad t \in[n h,(n+1) h), \quad s \in[j h,(j+1) h), \quad n \geqslant n_{0}, \quad j \in\{1,2,3, \ldots\}
\end{aligned}
$$

and after some simplification we obtain

$$
x(n+1) \leqslant \frac{1}{1+a(n) h} x(n)+\frac{b(n) h}{1+a(n) h} \sum_{j=1}^{\infty} \mathcal{K}(j) x(n-j), \quad n \geqslant n_{0}
$$

where we let $[t / h]=n,[s / h]=j$ and $f(n)=f(n h)$. We note that for practical treatment of (3.19) for instance in computer simulations and numerical solutions, the form of $\mathcal{K}(\cdot)$ can be chosen appropriately. Nonetheless, as far as our analysis is concerned the discrete delay kernel $\mathcal{K}(\cdot)$ will have certain properties which we shall mention later on.

In the following we provide results for the discrete Halanay-type inequality with distributed delays.

THEOREM 3.2. Let $h>0$ and let $x(\cdot)$ be a nonnegative sequence satisfying

$$
\begin{aligned}
x(n+1) & \leqslant \frac{1}{1+a(n) h} x(n)+\frac{b(n) h}{1+a(n) h} \sum_{j=1}^{\infty} \mathcal{K}(j) x(n-j), \quad n \geqslant n_{0} \\
x(n) & =|\varphi(n)| \quad \text { for } n \in\left(-\infty, n_{0}\right]
\end{aligned}
$$

where the interval $n \in\left(-\infty, n_{0}\right]$ denotes $n \in\left\{\ldots, n_{0}-2, n_{0}-1, n_{0}\right\} ; \varphi(n)$ is a sequence defined for $n \in\left(-\infty, n_{0}\right]$ and $\sup _{n \in\left(-\infty, n_{0}\right)}|\varphi(n)|=M, M>0$; the parameters $a(n)$ and $b(n)$ defined for $n \in \mathbb{Z}$ denote nonnegative and bounded sequences; the delay kernel $\mathcal{K}(\cdot)$ is assumed to have the following properties

$$
\mathcal{K}(j) \in[0, \infty) \text { for } j \in\{1,2,3, \ldots\} \text { and } \sum_{j=1}^{\infty} \mathcal{K}(j) \lambda^{j}<\infty
$$


for some $\lambda>1$. Suppose

$$
a(n)-b(n) \sum_{j=1}^{\infty} \mathcal{K}(j) \geqslant \sigma, \quad n \in \mathbb{Z}
$$

where $\sigma=\inf _{n \in \mathbf{Z}}\left(a(n)-b(n) \sum_{j=1}^{\infty} \mathcal{K}(j)\right)>0$. Then there exists a real number $\tilde{\lambda}>1$ such that

$$
x(n) \leqslant\left(\sup _{j \in\left(-\infty, n_{0}\right]} x(j)\right)\left(\frac{1}{\tilde{\lambda}}\right)^{n-n_{0}} \quad \text { for } n>n_{0} .
$$

PROOF: From our hypothesis in (3.22) we can find a critical value $\lambda^{*}>1$ for which

$$
\sum_{j=1}^{\infty} \mathcal{K}(j) \lambda^{j+1}<\infty \text { for } 1<\lambda<\lambda^{*}
$$

For example, let $\mathcal{K}(j)=e^{-j}$ for $j \in\{1,2,3, \ldots\}$ and so

$$
\sum_{j=1}^{\infty} e^{-j} \lambda^{j+1}=\lambda \sum_{j=1}^{\infty}\left(\frac{\lambda}{e}\right)^{j}<\infty \quad \text { for } 1<\lambda<\lambda^{*}=e .
$$

Along with this observation we define the function $G$ by

$$
G(n, \lambda)=\frac{\lambda}{1+a(n) h}+\frac{b(n) h}{1+a(n) h} \sum_{j=1}^{\infty} \mathcal{K}(j) \lambda^{j+1}-1, \quad n \in \mathbb{Z}, \quad 0<\lambda<\lambda^{*} .
$$

Since the sequences $a(n)$ and $b(n)$ are nonnegative and bounded for $n \in \mathbb{Z}$ we consider

$$
F(\lambda)=\sup _{n \in \mathbf{Z}} G(n, \lambda), \quad 0<\lambda<\lambda^{*} .
$$

Clearly, $F$ is continuous and defined for $0<\lambda<\lambda^{*}$. By applying the assumption (3.23) we have

$$
\begin{aligned}
F(1) & =\sup _{n \in \mathbf{Z}}\left(\frac{1}{1+a(n) h}+\frac{b(n) h}{1+a(n) h} \sum_{j=1}^{\infty} \mathcal{K}(j)-1\right) \\
& =-\inf _{n \in \mathbf{Z}}\left(\frac{h\left[a(n)-b(n) \sum_{j=1}^{\infty} \mathcal{K}(j)\right]}{1+a(n) h}\right) \leqslant-\frac{\sigma h}{1+a^{*} h}<0
\end{aligned}
$$

since $h>0, \sigma>0$ and $a^{*}>0$. Moreover,

$$
F_{*}(\lambda) \leqslant F(\lambda) \leqslant F^{*}(\lambda) \text { for } 0<\lambda<\lambda^{*}
$$


where

$$
\begin{array}{r}
F_{*}(\lambda)=\frac{\lambda}{1+a^{*} h}+\frac{b_{*} h}{1+a^{*} h} \sum_{j=1}^{\infty} \mathcal{K}(j) \lambda^{j+1}-1, \quad 0<\lambda<\lambda^{*} \\
F^{*}(\lambda)=\frac{\lambda}{1+a_{*} h}+\frac{b^{*} h}{1+a_{*} h} \sum_{j=1}^{\infty} \mathcal{K}(j) \lambda^{j+1}-1, \quad 0<\lambda<\lambda^{*} \\
a_{*}=\inf _{n \in \mathbf{Z}} a(n), \quad a^{*}=\sup _{n \in \mathbf{Z}} a(n), \quad b_{*}=\inf _{n \in \mathbf{Z}} b(n), \quad b^{*}=\sup _{n \in \mathbf{Z}} b(n) .
\end{array}
$$

We observe that $F_{*}(\lambda) \rightarrow \infty, F^{*}(\lambda) \rightarrow \infty$ as $\lambda \rightarrow \lambda_{-}^{*}$ and hence it follows from (3.29) that

$$
F(\lambda)=\sup _{n \in \mathbf{Z}} G(n, \lambda) \rightarrow \infty \quad \text { as } \quad \lambda \rightarrow \lambda_{-}^{*} .
$$

Let $0<\tilde{\sigma}<\sigma$. From (3.28), (3.30) and the continuity of $F(\lambda)$ there exists a number $\tilde{\lambda}$ satisfying $1<\tilde{\lambda}<\lambda^{*}$ such that

$$
F(\widetilde{\lambda})=\sup _{n \in \mathbf{Z}}\left(\frac{\tilde{\lambda}}{1+a(n) h}+\frac{b(n) h}{1+a(n) h} \sum_{j=1}^{\infty} \mathcal{K}(j) \tilde{\lambda}^{j+1}-1\right) \leqslant-\frac{\tilde{\sigma} h}{1+a^{*} h}<0
$$

and this in turn implies that

$$
\frac{\tilde{\lambda}}{1+a(n) h}+\frac{b(n) h}{1+a(n) h} \sum_{j=1}^{\infty} \mathcal{K}(j) \tilde{\lambda}^{j+1} \leqslant 1-\frac{\tilde{\sigma} h}{1+a^{*} h}=\rho \text { for all } n \in \mathbb{Z}
$$

where the constant $\rho$ satisfies $0<\rho<1$ since $0<\tilde{\sigma}<\sigma<a^{*}$.

Now we define

$$
\widetilde{x}(n)= \begin{cases}x(n) \tilde{\lambda}^{n-n_{0}}, & n>n_{0} \\ x(n), & -\infty<n \leqslant n_{0} .\end{cases}
$$

We have from (3.32) and (3.20),

$$
\begin{array}{rlrl}
\widetilde{x}(n+1) & =x(n+1) \tilde{\lambda}^{n+1-n_{0}}, \quad n \geqslant n_{0} & \\
& \leqslant\left(\frac{1}{1+a(n) h} x(n)+\frac{b(n) h}{1+a(n) h} \sum_{j=1}^{\infty} \mathcal{K}(j) x(n-j)\right) \tilde{\lambda}^{n+1-n_{0}}, \quad & & n \geqslant n_{0} \\
& =\frac{\tilde{\lambda}}{1+a(n) h} \widetilde{x}(n)+\frac{b(n) h}{1+a(n) h} \sum_{j=1}^{\infty} \mathcal{K}(j) \widetilde{\lambda}^{j+1} \widetilde{x}(n-j), & & n \geqslant n_{0} .
\end{array}
$$

Let $\delta>1$ be arbitrary. We have from (3.21) and (3.32) that $\widetilde{x}(n)<\delta M$ for $n \in\left(-\infty, n_{0}\right]$. We claim

$$
\widetilde{x}(n)<\delta M \text { for } n>n_{0} .
$$


Suppose (3.34) does not hold. Let the integer $n_{1}>n_{0}$ (the first time) be such that

$$
\widetilde{x}(n)<\delta M, \quad-\infty<n<n_{1} \text { and } \tilde{x}\left(n_{1}\right) \geqslant \delta M \text {. }
$$

We have from (3.33) and (3.35),

$$
\begin{aligned}
\delta M \leqslant \tilde{x}\left(n_{1}\right) \leqslant & \frac{\tilde{\lambda}}{1+a\left(n_{1}-1\right) h} \tilde{x}\left(n_{1}-1\right) \\
& \quad+\frac{b\left(n_{1}-1\right) h}{1+a\left(n_{1}-1\right) h} \sum_{j=1}^{\infty} \mathcal{K}(j) \tilde{\lambda}^{j+1} \widetilde{x}\left(n_{1}-1-j\right) \\
\leqslant & \frac{\tilde{\lambda}}{1+a\left(n_{1}-1\right) h} \tilde{x}\left(n_{1}-1\right) \\
& \quad+\frac{b\left(n_{1}-1\right) h}{1+a\left(n_{1}-1\right) h}\left(\sum_{j=1}^{\infty} \mathcal{K}(j) \tilde{\lambda}^{j+1}\right)\left(\sup _{s \in\left(-\infty, n_{1}-2\right]} \widetilde{x}(s)\right) \\
< & \left(\frac{\tilde{\lambda}}{1+a\left(n_{1}-1\right) h}+\frac{b\left(n_{1}-1\right) h}{1+a\left(n_{1}-1\right) h} \sum_{j=1}^{\infty} \mathcal{K}(j) \tilde{\lambda}^{j+1}\right) \delta M \\
\leqslant & \rho \delta M<\delta M \quad(\text { due to }(3.31))
\end{aligned}
$$

which leads to a contradiction. Hence the claim (3.34) holds. Since $\delta>1$ is arbitrary, by allowing $\delta \rightarrow 1_{+}$we have $\widetilde{x}(n) \leqslant M$ for $n>n_{0}$. It follows from (3.32) that $x(n) \leqslant$ $M(1 / \tilde{\lambda})^{n-n_{0}}$ for $n>n_{0}$ and hence the assertion (3.24) is satisfied. This completes the proof.

We conclude with a remark that the results obtained in this article can be used for the derivation of sufficient conditions for the existence of (uniformly asymptotically stable) periodic and almost periodic solutions of certain nonlinear scalar systems. These applications will be considered elsewhere.

\section{REFERENCES}

[1] R.P. Agarwal, Difference equations and inequalities: Theory, methods and applications (Marcel Dekker Inc., New York, 1992).

[2] C.T.H. Baker and A. Tang, 'Generalised Halanay inequalities for Volterra functional differential equations and discretised versions', Numerical Analysis Report 229, (Manchester Center for Computational Mathematics, University of Manchester, England, 1996).

[3] K.L. Cooke and I. Györi, 'Numerical approximation of the solutions of delay differential equations on an infinite interval using piecewise constant arguments', Comp. Math. Appl. 28 (1994), 81-92.

[4] K. Gopalsamy, Stability and oscillations in delay differential equations of population dynamics (Kluwer Academic Publishers, Dordrecht, The Netherlands, 1992).

[5] A. Halanay, Differential equations: stability, oscillations, time lags (Academic Press, New York, 1966). 
[6] V. Lakshmikantham and S. Leela, Differential and integral inequalities: Theory and applications. Vol. I: Ordinary differential equations (Academic Press, New York, 1969).

[7] V. Lakshmikantham and S. Leela, Differential and integral inequalities: Theory and applications. Vol. II: Functional, partial, abstract, and complex eifferential equations (Academic Press, New York, 1969).

[8] W. Walter, Differential and integral inequalities (Springer-Verlag, Berlin, Heidelberg, New York, 1970).

School of Informatics and Engineering,

Flinders University of South Australia,

Bedford Park SA 5042

Australia

e-mail: gopal@ist.flinders.edu.au 